\title{
A Variational Approach for an Electron Interacting with an Impenetrable Dielectric Surface under the Effect of an External Magnetic Field
}

\author{
B. SAQQA \\ Department of Physics, The Islamic University of Gaza, P.O. Box 108, Gaza Strip, Palestinian Authority
}

(Received November 16, 2008; in final form May 4, 2010)

\begin{abstract}
Using a variational approach, the interaction of an extrinsic electron with the surface modes of a semi-infinite medium is studied under the effect of an external magnetic field. The approach is to be valid for all values of the electron-surface-phonon coupling. In addition to the ground state energy, the number of phonons around the electron, and the spatial localization of the electron perpendicular and parallel to the surface are studied. It is observed that the magnetic field enhances the effective electron-phonon coupling and thus leads to an increased degree of localization of the electron toward the surface.
\end{abstract}

PACS: $71.38-\mathrm{k}$

\section{Introduction}

The problem of an electron interacting with the surface elementary excitations on a semi-infinite medium has been the subject of considerable interest in the past decades [1-10]. This interaction is interesting from a technological viewpoint in the context of surface spectroscopy and the study of the optical properties of polar thin films and interfaces.

Evans and Mills [1] used the Lee-Low-Pines theory to study the binding of electrons to the crystal surface. They considered the cases where the electron is outside and inside the crystal. Tokuda [2], and Bodas and Hipolito [3] considered only the case where the electron lies outside the crystal. They observed a phase-transition-like behavior from the quasi-free to the self-trapping electron state as the electron-phonon coupling constant exceeds a certain critical value. The effect of the magnetic field on the problem is investigated by Bhattacharya et al. [4] using a canonical transformations method. Using a variational approach, Ninno and Iadonisi [5] calculated the ground state energy and the wave function of an electron near the surface of a polar crystal. Xiao et al. [6] studied the properties of the surface polaron in polar crystals. Saqqa et al. [7] and Xiukun [8] studied the effect of the magnetic field on an electron near the surface of a crystal using the strong-coupling theory. The effect of both the magnetic field and the temperature is addressed by Eerdunchaolu et al. [9]. Maslov et al. [10] studied the effect of surface polaron in quantum dots.

The common conclusion reached by the previous mentioned works is that for a sufficiently large coupling constant the electron goes into a bound state in which it is localized in the close vicinity of the exterior face of the material by the strong interaction with the surface os- cillation modes. It has been observed that certain polaron quantities such as the ground state energy, the mean number of phonons around the electron, or the degree of localization are all enhanced by coupling strength. A further important finding is that the effective potential deviates considerably from the classical Coulomb profile $\left(V_{\text {class }} \propto-1 / z\right)$ and in particular, at distances close to the surface, the electron-phonon coupling imposes a rounding off of the divergence encountered in the classical picture. The application of an external magnetic field in the problem brought about an additional contribution to the localization of the electron so that the electron interacts with the phonons in a more efficient manner. This leads to an enhancement in the binding and an increase in the degree of confinement of the electron toward the surface.

In the following it is referred to the surface polaron problem considering the case where the electron is outside the medium and the effect of an external magnetic field using the variational method of Devreese et al. [11] is studied. The procedure is a combination between the adiabatic approximation and the first order perturbation method by adopting a variational trial function by which it is possible to extrapolate from the strong coupling regime toward the weak coupling one.

The model adopted in this work consists of a ionic or polar material filling the half space $z<0$ and an electron localized near, but completely external to, the material surface. The exterior electron has an electric field which influences and polarizes the surface modes. These modes, when polarized, create electric fields which in turn act back upon the electron. The electron is therefore attracted to the surface $(z=0)$ by its image potential and in the mean time is repelled away by the repulsive barrier resulting from the large difference between the 
bottom of the conduction band and the vacuum level of the material. It should be noted that the interactions of the electron with the bulk modes are ignored since the electron is outside the material.

In a previous work [12] we calculated only the ground state energy. In this report in addition to the ground state energy, we study the effective potential, the effective mass, and the spatial localization of the electron perpendicular to the surface.

\section{Theory}

Using the symmetric gauge $\boldsymbol{A}=(B / 2)(-y, x, 0)$ for the vector potential, the Hamiltonian describing an electron with position $\boldsymbol{r}=(\boldsymbol{\rho}, z)$ coupled to the surface optical (SO) phonons and acted upon by a uniform magnetic field perpendicular to the surface can be written as

$$
\begin{aligned}
& H=H_{0}+\sum_{\kappa} a_{\kappa}^{\dagger} a_{\kappa}+\sum_{\kappa} \Gamma_{\kappa}\left(a_{\kappa} \mathrm{e}^{\mathrm{i} \boldsymbol{\kappa} \cdot \boldsymbol{\rho}}+a_{Q}^{\dagger} \mathrm{e}^{\mathrm{i} \boldsymbol{\kappa} \cdot \boldsymbol{\rho}}\right), \\
& H_{0}=p_{x}^{2}+p_{y}^{2}+\frac{1}{4}(\Omega / 2)^{2}\left(x^{2}+y^{2}\right)+(\Omega / 2) l_{z},
\end{aligned}
$$

where the operators $a_{\kappa}^{\dagger}$ and $a_{\kappa}$ are the creation and annihilation operators of a phonon of wave vector $\kappa$, respectively, and $\Omega$ is the dimensionless cyclotron frequency. The interaction amplitude is related to the electron-SO-phonons coupling constant $\alpha$ and the phonon wave vector $\boldsymbol{\kappa}$ through the relation [1]:

$$
\Gamma_{\kappa}=\sqrt{\frac{2 \pi \alpha}{S \kappa}} \mathrm{e}^{-\kappa z},
$$

where $S$ is the normalization area. $L_{z}$ in Eq. (2) is the $z$-component of the angular momentum. It should be noted that all physical quantities and operators will be given in dimensionless form where energies are scaled by the SO-phonon $\hbar \omega$, and lengths by the polaron radius $\sqrt{\hbar / 2 m \omega}$.

The procedure followed here starts from the adiabatic approach where the electron and the lattice variables are assumed to be totally separable, that is, the wave function of the problem will be given by

$$
\Psi=\Phi_{\mathrm{e}} \Phi_{\mathrm{ph}},
$$

where $\Phi_{\mathrm{e}}$ represents the electronic part of the wave function. The phonon part $\Phi_{\mathrm{ph}}$ is given as

$$
\Phi_{\mathrm{ph}}=\mathrm{e}^{S}|0\rangle,
$$

where $|0\rangle$ is the phonon vacuum. The exponential operator with

$$
S=\sum_{\kappa} F_{\kappa}\left(a_{\kappa}-a_{\kappa}^{\dagger}\right)
$$

is the canonical coherent state transformation which, in the semiclassical approach, leads to the optimal surface polarization centered on the mean charge density induced, by electron, on the surface of the material. The amplitude $F_{\kappa}$ depends implicitly on the electron wave function and must be treated as a variational parameter to be determined by the requirement that the energy of the system be minimized. Optimization of the transformed Hamiltonian

$$
\begin{aligned}
H^{\prime} & =\mathrm{e}^{-S} H \mathrm{e}^{S}=H_{0}+\sum_{\kappa} a_{\kappa}^{\dagger} a_{\kappa}+\sum_{\kappa} F_{\kappa}^{2} \\
& -\sum_{\kappa} F_{\kappa}\left(a_{\kappa}+a_{\kappa}^{\dagger}\right)+\sum_{\kappa} \Gamma_{\kappa}\left(a_{\kappa} \mathrm{e}^{\mathrm{i} \boldsymbol{\kappa} \cdot \boldsymbol{\rho}}+a_{\kappa}^{\dagger} \mathrm{e}^{-\mathrm{i} \boldsymbol{\kappa} \cdot \boldsymbol{\rho}}\right) \\
& -\sum_{\kappa} \Gamma_{\kappa} F_{\kappa}\left(\mathrm{e}^{\mathrm{i} \boldsymbol{\kappa} \cdot \boldsymbol{\rho}}+\mathrm{e}^{-\mathrm{i} \boldsymbol{\kappa} \cdot \boldsymbol{\rho}}\right) .
\end{aligned}
$$

With respect to the ground state $\Phi_{\mathrm{e}}|0\rangle$ we obtain

$$
F_{\kappa}=\left\langle\Phi_{\mathrm{e}}\left|\Gamma_{\kappa} \mathrm{e}^{ \pm \mathrm{i} \boldsymbol{\kappa} \cdot \boldsymbol{\rho}}\right| \Phi_{\mathrm{e}}\right\rangle,
$$

and the ground state energy in the strong coupling regime is given by

$$
E_{\mathrm{sc}}=e_{0}-e_{\mathrm{p}}=\left\langle\Phi_{\mathrm{e}}\left|H_{0}\right| \Phi_{\mathrm{e}}\right\rangle-\sum_{\kappa} F_{\kappa}^{2} .
$$

To extend the formulation to the weak coupling case, we include a first order correction to the trial state $\Phi_{\mathrm{e}}|0\rangle$ with the last term in Eq. (7) being a perturbation. We then have

$$
\delta \Psi=\sum_{\kappa} \sum_{j} \frac{|j\rangle\left\langle j\left|\eta_{\kappa}^{*} a_{\kappa}^{\dagger}\right| \Phi_{\mathrm{e}}\right\rangle|0\rangle}{\varepsilon_{0}-\varepsilon_{j}-1},
$$

where

$$
\eta_{\lambda}=\Gamma_{\kappa} \mathrm{e}^{\mathrm{i} \kappa \cdot \rho}-F_{\kappa} .
$$

The summation over the intermediate states can be projected out simply by replacing the energy dominator by an average quantity which in the calculation will be determined variationally. Using completeness we can write

$$
\delta \Psi=\sum_{\kappa} \Gamma_{\kappa} g_{\kappa} \eta_{\kappa}^{*} a_{\kappa}^{\dagger}\left|\Phi_{\mathrm{e}}\right\rangle|0\rangle,
$$

where $g_{\kappa}$ is another variational parameter. The trial wave function of the Hamiltonian $H^{\prime}$ is then extended to

$$
\Psi^{\prime}=n \Phi_{\mathrm{e}}|0\rangle+\delta \Psi,
$$

where $n$ is normalization constant. The optimal fit to $g_{\kappa}$ is achieved by minimizing $\left\langle\Psi^{\prime}\left|H^{\prime}\right| \Psi^{\prime}\right\rangle$ subject to the constraint

$$
\left\langle\Psi^{\prime} \mid \Psi^{\prime}\right\rangle=n^{2}\left(1+\sum_{\kappa} \Gamma_{\kappa}^{2}\left(g_{\kappa} / n\right)^{2} h_{\kappa}\right)=1,
$$

in which

$$
h_{\kappa}=\left\langle\Phi_{\mathrm{e}}\left|\eta_{\kappa} \eta_{\kappa}^{*}\right| \Phi_{\mathrm{e}}\right\rangle \text {. }
$$

For the energy we then have

$$
E_{\mathrm{g}}=E_{\mathrm{sc}}+\lambda \text {, }
$$

where $\lambda$ is a Lagrange multiplier depending on $\alpha$ and $\Omega$ through the equation

$$
\lambda=\sum_{\kappa} \Gamma_{\kappa}^{2}\left(g_{\kappa} / n\right) h_{\kappa},
$$

where with

$$
g_{\kappa} / n=-h_{\kappa} /\left[e_{\kappa}-f_{\kappa}+\left(1-\lambda-e_{0}-e_{\mathrm{p}}\right) h_{\kappa}\right],
$$

$$
e_{\kappa}=\left\langle\Phi_{\mathrm{e}}\left|\eta_{\kappa} H_{0} \eta_{\kappa}^{*}\right| \Phi_{\mathrm{e}}\right\rangle
$$

and

$$
f_{\kappa}=\sum_{\kappa^{\prime}} \Gamma_{\kappa^{\prime}}^{2} F \kappa^{\prime}\left\langle\Phi_{\mathrm{e}}\left|\eta_{\kappa}\left(\mathrm{e}^{\mathrm{i} \kappa \cdot \rho}+\mathrm{e}^{-\mathrm{i} \kappa \cdot \rho}\right) \eta_{\kappa^{\prime}}^{*}\right| \phi_{\mathrm{e}}\right\rangle .
$$

Selecting a Gaussian spread (with variance $\sigma^{2}$ ) in the transverse direction and describing the localization in the 
remaining direction by

$$
\Phi_{z}=\left(\beta^{2} / 2\right)^{1 / 2} z \mathrm{e}^{-\beta z / 2},
$$

with $\beta$ to be determined variationally, we obtain

$$
\begin{aligned}
& e_{0}=\sigma^{-2}+\frac{1}{4} \beta^{2}+\left(\frac{1}{4} \Omega \sigma\right)^{2}, \\
& e_{\mathrm{p}}=\alpha \int_{0}^{\infty} \mathrm{d} \kappa F_{\kappa}^{2}, \\
& F_{\kappa}=S_{\kappa}(\sigma) R_{\kappa}(\beta)=\mathrm{e}^{-\left(\frac{1}{4} \sigma^{2} \kappa^{2}\right)}\left(1+\frac{\kappa}{\beta}\right)^{-3}, \\
& h_{\kappa}=R_{2 \kappa}(\beta)-S_{\kappa}^{2}(\sigma) R_{\kappa}^{2}(\beta), \\
& e_{\kappa}=R_{2 \kappa}(\beta)\left[e_{0}+2 \kappa^{2}+\kappa \beta\right] \\
& \quad+S_{\kappa}^{2}(\sigma) R_{\kappa}^{2}(\beta)\left[e_{0}-2 \sigma^{-2}-\frac{1}{2}(\kappa+\beta)^{2}\right], \\
& f_{\kappa}=2 \alpha \int_{0}^{\infty} \mathrm{d} \kappa^{\prime} S_{\kappa^{\prime}}^{2} R_{\kappa^{\prime}}\left[R_{2 \kappa+\kappa^{\prime}}+S_{\kappa}^{2} R_{\kappa}^{2} R_{\kappa^{\prime}}\right] \\
& \quad-4 \alpha S_{\kappa}^{2} R_{\kappa} \int_{0}^{\infty} \mathrm{d} \kappa^{\prime} S_{\kappa^{\prime}}^{2} R_{\kappa^{\prime}} R_{\kappa+\kappa^{\prime}} I_{0}\left(\kappa \kappa^{\prime} \sigma^{2} / 2\right),
\end{aligned}
$$

in which $I_{0}$ is the modified Bessel function of order zero.

To calculate the mean number of phonons in the cloud around the electron we have

$$
\langle n\rangle=\left\langle\Psi^{\prime}\left|\mathrm{e}^{-S} \sum_{\kappa} a_{\kappa}^{\dagger} a_{\kappa} \mathrm{e}^{S}\right| \Psi^{\prime}\right\rangle .
$$

After performing a straightforward calculation we obtain

$$
\langle n\rangle=\frac{b}{1+b}-e_{\mathrm{p}}
$$

with

$$
b=\sum_{\kappa} \Gamma_{\kappa}^{2}\left(g_{\kappa} / n\right)^{2} h_{\kappa}=\alpha \int_{0}^{\infty}\left(g_{\kappa} / n\right)^{2} h_{q} \mathrm{~d} q .
$$

The localization of the polaron in a direction perpendicular to the surface can be found by computing the mean value of $z$-coordinate, that is

$$
\begin{aligned}
& \left\langle z^{2}\right\rangle=12 \frac{n^{2}}{\beta^{2}}\left\{1+\sum_{\kappa} \Gamma_{\kappa}^{2}\left(g_{\kappa} / n\right)^{2}\left[\left(1+2 \kappa \beta^{-1}\right) R_{2 \kappa}^{2}\right.\right. \\
& \left.\left.-2 F_{\kappa}^{2} R_{\kappa}\left(1+\kappa \beta^{-1}\right)+F_{\kappa}^{2}\right]\right\} .
\end{aligned}
$$

In the same manner, the localization of the electron in a direction parallel to the surface can be found by computing the mean value of the $\rho$-coordinate, that is

$$
\left\langle\rho^{2}\right\rangle=\frac{2}{\sigma}+2 \frac{n^{2}}{\sigma^{2}} \sum_{\kappa} \Gamma_{\kappa}^{2}\left(g_{\kappa} / n\right)^{2} \kappa^{2} F_{\kappa}^{2} .
$$

\section{Results and discussion}

Before discussing the numerical results of the present approach, we want to test the theory for some limiting cases. For large values of the coupling constant $\alpha, S_{\kappa}(\sigma)$ and $R_{\kappa}(\beta)$, defined by Eq. (24), both approach unity and hence $\lambda \rightarrow 0$. This means that the ground state energy defined in Eq. (16) conforms to that given by Eq. (9), which is the strong coupling limit. For small $\alpha$, however, the role of $\lambda$ becomes very prominent and the polaron binding is dominantly determined by this term. If the charge of the electron is restricted to be fluctuating just on the surface, the present model becomes similar to that for strictly two-dimensional magnetopolaron [14]. Imposing an infinitely large value for $\beta$ in Eq. (21), the strong coupling ground state energy simplifies to

$$
E_{\mathrm{g}}=\sigma^{-2}+\left(\frac{1}{4} \Omega \sigma\right)^{2} \sqrt{\frac{\pi}{2}} \alpha \sigma^{-1} .
$$

For strong coupling and weak magnetic field $\left(\Omega / \alpha^{2} \ll 1\right)$, the dominant contribution comes from the first and the third terms in Eq. (31). Minimization of the dominant part gives

$$
E_{\mathrm{g}}=-\frac{\pi}{8} \alpha^{2}\left[1-\left(2 \Omega / \pi \alpha^{2}\right)^{2}\right],
$$

which is the two-dimensional analogue of the corresponding bulk value [14].

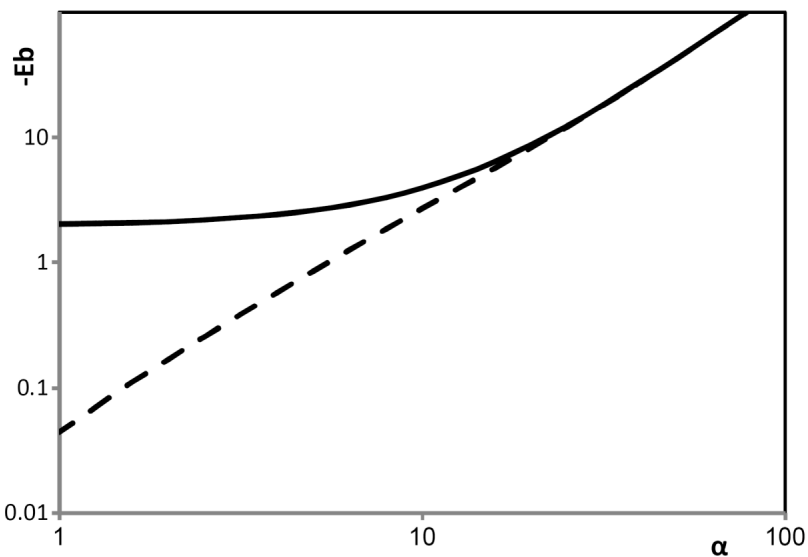

Fig. 1. The binding energy as a function of $\alpha$ for $\Omega=0$ (solid line) and $\Omega=4$ (dashed line).

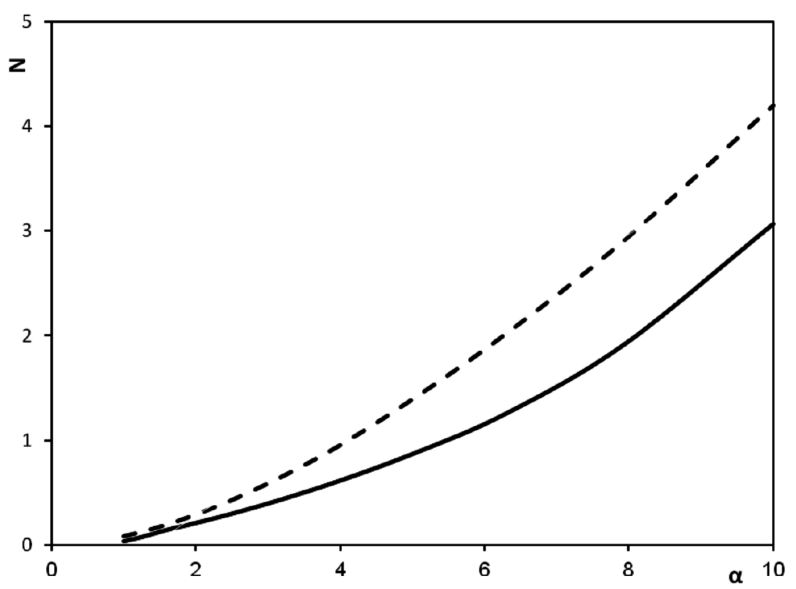

Fig. 2. The number of phonons around the electron as a function of $\alpha$ for $\Omega=0$ (solid line) and $\Omega=4$ (dashed line). 


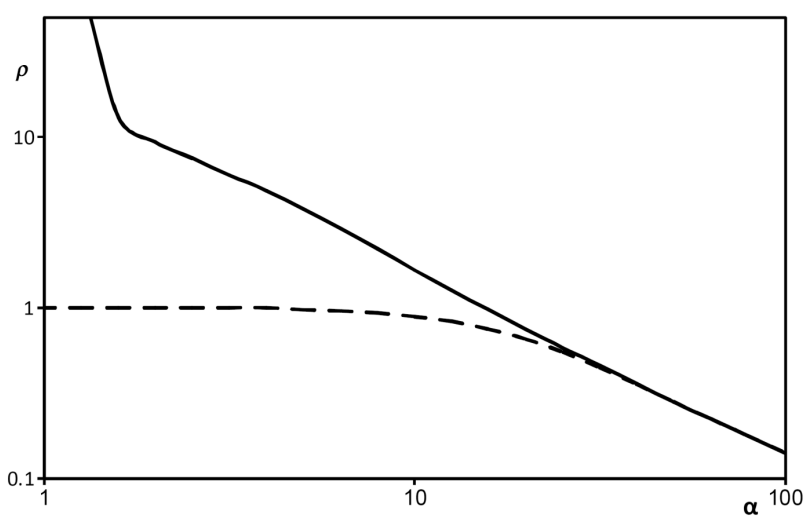

Fig. 3. The mean square root of the transverse extent as a function of $\alpha$ for $\Omega=0$ (solid line) and $\Omega=4$ (dashed line).

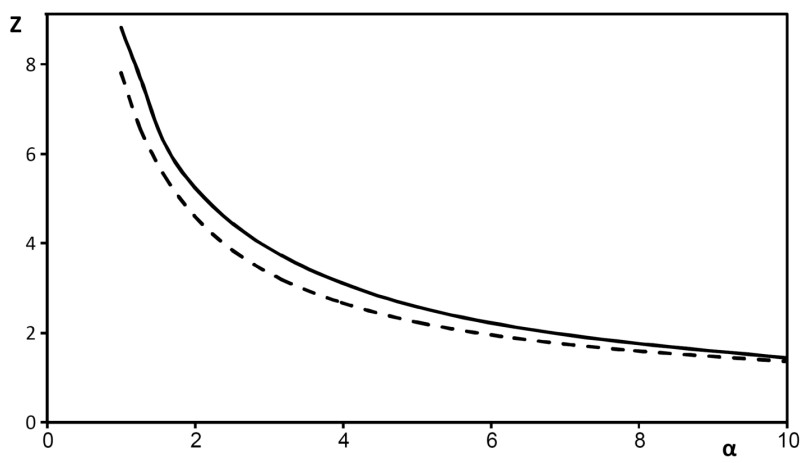

Fig. 4. The mean square root of the distance from the surface as a function of $\alpha$ for $\Omega=0$ (solid line) and $\Omega=4$ (dashed line).

To study the problem for all values of the coupling constant $\alpha$, and in the overall range of the magnetic field, we minimize Eq. (16) by numerical techniques. Figure 1 shows the dependence of the binding energy $\left(E_{\mathrm{b}}=E_{\mathrm{g}}-\frac{1}{2} \Omega\right)$ on $\alpha$ with $(\Omega=4)$ and without $(\Omega=0)$ the magnetic field. From the figure we conclude that for reasonable values $\alpha(\alpha<10)$, the magnetic field enhances the effect of the polaronic correction to the binding energy. For large values of $\alpha$, however, the magnetic field loses its effect on the problem due to the dominancy of the polaronic field.

In Fig. 2 we display the number of phonons around the electron as a function of $\alpha$, again without and with the effect of the magnetic field. As it is clear from the figure the number of phonons increases with $\alpha$. Moreover, the magnetic field has a role in increasing the number of phonons. This is due to the fact that the magnetic field increases the localization of the electron toward the surface and this increases the importance of the electronphonon interaction.

In Fig. 3 we plot the mean square root of the transverse extent versus $\alpha$ for $\Omega=0$ and $\Omega=4$. In the absence of the magnetic field we observe that for weak coupling the electron becomes free to move parallel to the surface which means that the interaction of the electron with the SO-phonons loses its importance to a large extent in this limit. When the magnetic field is turned on, the coupling of the electron to the surface phonon modes becomes more enhanced.

The same phenomenon shows up when we plot the mean square root of the distance from the surface versus $\alpha$ for $\Omega=0$ and $\Omega=4$ (Fig. 4). Again the spatial localization of the electron perpendicular to the surface shrinks as $\alpha$ is getting smaller. The magnetic field pulls the electron toward the surface a little bit.

\section{Conclusion}

In this report we have retrieved the problem of an electron interacting with the surface polaron under the effect of an external magnetic field using a variational approach intended to be valid for the overall range of the coupling constant. The ground state energy, the number of phonons around the electron, and the localization of the polaron perpendicular and parallel to the surface have been calculated. It is found that the magnetic field enhances the polaronic effect due to the additional confinement brought about by the magnetic field.

\section{References}

[1] E. Evans, D.L. Mills, Phys. Rev. B 8, 4004 (1973).

[2] N. Tokuda, J. Phys. C, Solid State Phys. 15, 1353 (1982).

[3] E.L. Bodas, O. Hipolito, Phys. Rev. B 27, 6110 (1983).

[4] M. Bhattacharya, S. Mukhopadhyay, T.K. Mitra, Phys. Status Solidi B 142, 141 (1987).

[5] D. Ninno, G. Iadonisi, Phys. Rev. B 38, 3808 (1988).

[6] J.L. Xiao, T.H. Xing, B.Q. Sun, W. Xiao, Phys. Status Solidi B 183, 425 (1994).

[7] B. Saqqa, R.T. Senger, A. Erçelebi, Turk. J. Phys. 20, 1062 (1996).

[8] H. Xiukun, arXiv: cond-mat/0311061 V1, 1 (2003).

[9] Eerdunchaolu, W. Xiao, J.L. Xiao, Int. J. Mod. Phys. B; Condens. Matter Phys. 18, 2003 (2004).

[10] A.Yu. Maslov, O.V. Proshina, A.N. Rusina, in: 28th Int. Conf. on the Physics of Semiconductors - ICPS 2006, DOI: 10. 1063/1.2730174, 2007.

[11] J. Devreese, R. Evrard, E. Kartheuser, F. Brosens, Solid State Commun. 44, 1435 (1982).

[12] B. Saqqa, J. Al-Aqsa Univ. 10, 238 (2006).

[13] W. Xiaoguang, F.M. Peeters, J.T. Devreese, Phys. Rev. B 32, 7964 (1985).

[14] Y. Lépine, J. Phys. C, Solid State Phys. 18, 1817 (1985). 